\title{
Characteristics of Novel Chicken Embryonic Stem Cells Established Using Chicken Leukemia Inhibitory Factor
}

\author{
Mikiharu Nakano ${ }^{1}$, Kenjiro Arisawa ${ }^{2}$, Satomi Yokoyama ${ }^{1}$, Masaki Nishimoto ${ }^{2}$, Yusuke Yamashita ${ }^{1}$, \\ Maya Sakashita ${ }^{1}$, Ryo Ezaki ${ }^{1}$, Haruo Matsuda ${ }^{1}$, Shuichi Furusawa ${ }^{1}$ and Hiroyuki Horiuchi ${ }^{1,2}$ \\ ${ }^{1}$ Department of Molecular and Applied Biosciences, Graduate School of Biosphere Science, \\ Hiroshima University, 1-4-4 Kagamiyama, Higashi-Hiroshima 739-8528, Japan \\ ${ }^{2}$ Hiroshima Prefectural Institute of Industrial Science and Technology, Hiroshima Industrial \\ Promotion Organization, 3-10-32 Kagamiyama, Higashi-Hiroshima 739-0046, Japan
}

\begin{abstract}
Chickens are an ideal animal model study tool for developmental biology, and a farm animal with excellent productivity. Researchers have therefore long sought to establish chicken embryonic stem cells (cESCs) to enable the creation of genetically modified chickens. Here, we derived novel cESCs from chicken blastodermal cells (CBCs) cultured with chicken leukemia inhibitory factor (chLIF). These cESCs have the capacity for long-term (100 days or more) successive subculture and express both chicken Nanog (chNanog) and chicken vasa homolog (Cvh) mRNAs and proteins. The cESCs showed a capacity for chimeric formation during a transplant experiment that used a fertilized egg. Transfer of the enhanced green fluorescent protein (EGFP) gene to cESCs enabled green fluorescence to be observed among primordial germ cells (PGCs). These results indicate that novel cESCs should have the capacity to differentiate into germ cells.
\end{abstract}

Key words: chicken embryonic stem cells, chicken Nanog, chicken vasa homolog, leukemia inhibitory factor, primordial germ cells

J. Poult. Sci., 48: 64-72, 2011

\section{Introduction}

In 1981, mouse embryonic stem (ES) cell lines were established by culturing the mouse inner cell mass (Evans and Kaufman, 1981; Martin, 1981). These mouse ES cells have an infinite proliferative capacity to differentiate into various cells derived from the ectoderm, mesoderm, and endoderm, both in vitro and in vivo. Genetically modified mice that are created from ES cells allow us to analyze the functions of specific genes in vivo, thereby contributing to the field of life science research. While attempts have been made to establish ES cells using animals other than mice, successful differentiation of ES cells into germ cells has only been achieved for mice (Evans and Kaufman, 1981; Martin 1981; Nichols et al., 1990), rats (Buehr et al., 2008; Li et al., 2008), and monkeys (Thomson and Marshall 1998; Suemori et al., 2001; Adachi et al., 2006; Teramura et al., 2007; Yamauchi et al., 2009).

During the early stages of chicken development, no cells

Received: October 12, 2010, Accepted: October 26, 2010

Released Online Advance Publication: December 25, 2010

Correspondence: H. Horiuchi, Ph, D., Department of Molecular and Applied Biosciences, Graduate School of Biosphere Science, Hiroshima University, 1-4-4 Kagamiyama, Higashi-Hiroshima 739-8528, Japan.

(E-mail: hhori10@hiroshima-u.ac.jp) correspond to the ICM of mammals. However, chicken blastodermal cells (CBCs), which derive from stage $\mathrm{X}$ embryo (Eyal-Giladi and Kochav, 1976) have the capacity to differentiate into all somatic cells and germ cells when transplanted into a recipient embryo of the same developmental stage (Petitte et al., 1990; Kagami et al., 1995). Thus, attempts have been made to establish chicken ES cells (cESCs) by incubating CBCs (Pain et al., 1996; van de Lavoir et al., 2006a). Unfortunately, after long-term culture, cESCs lose the expression of chicken vasa homolog $(\mathrm{Cvh})$ protein as the most specific marker for germ cells (Tsunekawa et al., 2000), as well as their capacity for differentiation into germ cells (van de Lavoir et al., 2006a, b).

Researchers have previously used leukemia inhibitory factor (LIF), isolated from mice or rats, to preserve the pluripotency of cESCs. However, our cloning of chicken LIF (chLIF) found it to share only 38\% identity with mouse LIF (mLIF) (Horiuchi et al., 2004). During the culture of CBCs, phosphorylation of signal transducer and activator of transcription 3 (STAT3) occurred in the presence of chLIF but not MLIF, while chLIF was shown to be more important than $\mathrm{mLIF}$ in preserving the pluripotency of cESCs (Horiuchi et al., 2004; Yamashita et al., 2006). 
In the present study, we report on the characteristics of novel cESCs that we successfully established using chLIF, as well as on the potential of creating genetically-modified chickens using ES cells.

\section{Materials and Methods}

\section{Experimental Animals, Tissues, and Cells}

White Leghorn (WL) and Barred Plymouth Rocks (BPR) chickens were maintained in an isolated facility at the University Animal Farm, Hiroshima University, Japan. Female New Zealand white rabbits (approximately $2.5 \mathrm{~kg}$ ) and 8-week old female BALB/c mice were purchased from Shimizu Laboratory Supplies Co. (Kyoto, Japan), and used to raise antibodies. Animals were maintained, bred, and used in experiments in accordance with Hiroshima University guidelines, and the study protocol was approved by its experimental animal committee (Authorization No. D07-19).

Freshly laid, fertilized, unincubated WL and BPR eggs were purchased from Akita Co. (Fukuyama, Japan) and incubated at $38^{\circ} \mathrm{C}$. Their embryonic stage was defined according to Eyal-Giladi and Kochav (1976) (shown as roman numerals) and Hamburger and Hamilton (1951) (shown as arabic numerals). CBCs were collected from stage $\mathrm{X}$ embryos as described previously (Horiuchi et al., 2006). Primordial germ cells (PGCs) were collected from the blood of stages $13^{-15}$ embryos (after about $50 \mathrm{~h}$ incubation) and concentrated by Ficoll density centrifugation as described previously (Tajima et al., 1993).

\section{Production of Recombinant chLIF}

The chick embryo cell line CHCC-OU2 (Ogura and Fujiwara, 1987), originally supplied by Dr. Fujiwara (Okayama University, Okayama, Japan), was routinely grown in low-glucose Dulbecco's modified Eagle's medium (DMEM) (Invitrogen, Carlsbad, CA) containing 10\% fetal bovine serum (FBS) (Hyclone; Thermo Fisher Scientific Inc, Walthan, MA), $100 \mu \mathrm{g} / \mathrm{m} l$ penicillin and $70 \mu \mathrm{g} / \mathrm{m} l$ streptomycin at $37^{\circ} \mathrm{C}$ in $5 \% \mathrm{CO}_{2}$. The coding region of chLIF was amplified using the following primers: sense primer for chLIF, GCGCTAGCCATGAGGCTCATCCCC; reverse primer for chLIF, GTCGACCGCGGGGCTGAGGTGAGGTA. PCR products were digested with Nhe I and Sal I and sub-cloned into the pSecTag2A plasmid (Invitrogen) containing the histidine tag. The myc-epitope was excised from the pSecTag2A plasmid using restriction enzymes. The recombinant plasmid was transfected into CHCC-OU2 cells using Polyfect Transfection Reagent (Qiagen, Hilden, Germany) and recombinant cells were selected in medium containing $0.25 \mu \mathrm{g} / \mathrm{m} l$ zeocin (Invitrogen). Several stable CHCC-OU2 cell lines secreting biologically active chLIF were selected and recombinant chLIF (rchLIF) was purified from culture supernatants on ProBond resin (Invitrogen).

\section{Incubation of Chicken ES Cells}

Sandoz inbred mouse-derived thioguanine-resistant and ouabain-resistant (STO) cells (American Type Cell Collection, Manassas, VA) were cultured to $80 \%$ confluence in low-glucose DMEM containing 10\% FBS. Feeder cells were prepared by treating STO cells with mitomycin C (Sigma-Aldrich, St. Louis, MO) and culturing them at a cell density of $1.5 \times 10^{4}$ cells $/ \mathrm{cm}^{2}$. The reason to select STO cells as feeder cells is to exclude contamination of the genes in chick-derived feeder cells when the expression of the genes in CBCs and cESCs is analyzed.

Using a filter paper ring, stage X CBCs were collected from one egg of BPR chickens as described previously (Horiuchi et al., 2006). CBCs were gently suspended using a micropipette, disseminated on a 12 -well plate, and cultured in a $\mathrm{CO}_{2}$ incubator. The chicken ES cell medium (CECM) contained high-glucose DMEM supplemented with 20\% KnockOut Serum Replacement (Invitrogen), $2 \%$ heat-inactived chicken serum (Invitrogen), $0.1 \mathrm{mM}$ sodium pyruvate, nucleocide $(8 \mu \mathrm{g} / \mathrm{m} l$ adenosine, $8.5 \mu \mathrm{g} /$ $\mathrm{ml}$ guanosine, $7.3 \mu \mathrm{g} / \mathrm{m} l$ cytidine, $7.3 \mu \mathrm{g} / \mathrm{m} l$ uridine, 2.4 $\mu \mathrm{g} / \mathrm{m} l$ thymidine), $0.05 \mathrm{mM}$ non-essential amino acid, streptomycin $(70 \mu \mathrm{g} / \mathrm{ml})$, penicillin $(100 \mu \mathrm{g} / \mathrm{ml}), 0.1 \mathrm{mM}$ $\beta$-mercaptoethanol and $\operatorname{rchLIF}(20 \mathrm{ng} / \mathrm{ml})$. Cultured cells were serially subcultivated every second or third day to ensure optimal culture and to inhibit differentiation. The cells were separated gently using the tip of a micropipette and disseminated onto a new plate. Half or all of the incubation medium was replaced daily with fresh medium. RT-PCR Analysis

Total RNA was extracted from cESCs using ISOGENLS (Nippon gene, Tokyo, Japan) according to the manufacturer's instructions. RNA was used for reverse transcription PCR (RT-PCR) with the SuperScript ${ }^{\mathrm{TM}}$ III FirstStrand Synthesis System for RT-PCR (Invitrogen) according to the manufacturer's instructions. PCR was carried out using the ABI PRISM 7700 sequence-detection system (Applied Biosystems) and SYBR Green core reagents (Applied Biosystems). The cDNA amounts were normalized to chicken GAPDH SYBR Green PCR, according to the manufacturer's instructions, and equal quantities were then used as templates in PCR reactions. PCR amplification was carried out with $0.4 \mu \mathrm{M}$ primers, $0.2 \mathrm{mM}$ dNTPs, $1 \mathrm{mM} \mathrm{MgCl} 2,10$ x Ex Taq buffer $\left(\mathrm{Mg}^{2+}\right.$ free) and $1 \mathrm{U} E x$ Taq (Takara) in a final volume of $25 \mu \mathrm{l}$ using a Gene Amp PCR System 9700 (Applied Biosystems). The PCR reaction consisted of 22,24 or 28 cycles (specified below) of $30 \mathrm{~s}$ at $94^{\circ} \mathrm{C}, 30 \mathrm{~s}$ at 58 or $60^{\circ} \mathrm{C}$, and $1 \mathrm{~min}$ at $72^{\circ} \mathrm{C}$. The PCR products were analyzed on $1.5 \%$ agarose gels and visualized with EtBr. The upstream and downstream primer pairs, annealing temperature and cycle numbers used for each gene were as follows: chNanog (TGACCACAGAGCAGAAAACG, CAGCCATGAACGGATACAGG, $58^{\circ} \mathrm{C}, 24$ cycles); Cvh (CAGCCATGAACGGATACAGG, ATGAATGTGCTGTTGAGATGTC, $60^{\circ} \mathrm{C}, 28$ cycles); GAPDH (GCACGCCATCACTATCTTCCAG, CGGCAGGTCAGGTCAACAACAG, $58^{\circ} \mathrm{C}, 22$ cycles). We confirmed that 22,24 or 28 PCR cycles represented the exponential phase of the amplification. Chicken GAPDH gene amplification products were used as internal controls. 


\section{Production of Specific Anti-chNanog and Anti-Cvh Anti- bodies}

Recombinant chNanog (rchNanog) and Cvh (rCvh), as antigens for antibody production, were expressed in the pMAL-c2X plasmid (New England Biolabs, Ipswich, MA) containing the maltose binding protein (MBP) gene and in the pGEX-6P-1 plasmid (GE Healthcare, Buckinghanshire, UK) containing the glutathione S-transferase (GST) gene, according to the manufacturer's instructions. The entire translated region of rchNanog was expressed and purified as MBP-rchNanog or GST-rchNanog fusion proteins. The partial translated region (amino acids 116464) of rCvh was expressed and purified as MBP-rCvh or GST-rCvh fusion proteins.

Anti-Cvh mouse monoclonal antibody $(\mathrm{mAb})$ was developed by immunizing 8 -week-old female BALB/c mice intraperitoneally with $50 \mu \mathrm{g}$ GST-rCvh mixed with an equal volume of complete Freund's adjuvant. After three bi-weekly boosts with the same dose of the immunogens in $0.1 \mathrm{~m} l \mathrm{PBS}$, mice with high serum titers of antibodies against MBP-rCvh were identified in enzyme-linked immunosorbent assays (ELISA). These mice were boosted intravenously with $50 \mu \mathrm{g}$ GST-rCvh in PBS and, three days later, their splenocytes were fused with SP2/0 Ag14 myeloma cells (Shulman et al., 1978) using established methods (Galfre et al., 1977). Hybridomas secreting specific antibodies were identified by ELISA (Yamashita et al., 2006), and cloned by limiting dilution. The immunoglobulin isotype of the mAb was determined using a mouse monoclonal antibody isotyping kit (GE Healthcare).

Anti-chNanog polyclonal antibody (pAb) was raised by immunizing female NZW rabbits subcutaneously with 300 $\mu \mathrm{g}$ MBP-rchNanog mixed with an equal volume of complete Freund's adjuvant. After four bi-weekly immunizations with the same dose of the immunogens, antisera from the rabbits were purified by affinity chromatography using GST-rchNanog-conjugated agarose beads and designated anti-chNanog pAb.

\section{Western Blot Analysis}

Nuclear and cytoplasmic extracts were prepared from freshly isolated CBCs, STO cells and chicken embryo fibroblast (CEF) using a Nuclear Extract Kit (Active Motif, Carlsbad, CA), according to the manufacturer's instructions. Testis and ovary lysates were prepared from newly hatched chicks using extraction buffer $(0.15 \mathrm{M}$ $\mathrm{NaCl}, 50 \mathrm{mM}$ Tris- $\mathrm{HCl}$ (pH7.4), $1 \mathrm{mM}$ EDTA, $0.1 \%$ sodium deoxycholate and $1 \%$ Nonidet P-40). One tablet of a complete protease-inhibitor cocktail (Roche, Mannheim, Germany) was added per $25 \mathrm{~m} l$ extraction buffer. Cell extracts or tissue extracts were separated on $10 \%$ SDS-PAGE gels under reducing conditions and transferred to PVDF membranes (Bio-Rad, Hercules, CA). Membranes were blocked with $20 \mathrm{mM}$ Tris- $\mathrm{HCl}, \mathrm{pH} 7.4$, $140 \mathrm{mM} \mathrm{NaCl}, 25 \mathrm{mM}$ EDTA, $0.2 \%$ Tween 20 and $4 \%$ nonfat milk overnight at $4^{\circ} \mathrm{C}$, washed three times in Tween-PBS, and incubated with Anti-Cvh mAb (hybrid- oma culture supernatants) diluted 1:5 or anti-chNanog $\mathrm{pAb}$ diluted 1:1,000 in blocking buffer containing 1\% nonfat milk (dilution buffer) for $1 \mathrm{~h}$ at room temperature (RT). After 3 washes as before, blots were incubated with horseradish peroxidase (HRP)-conjugated goat anti-mouse or anti-rabbit immunoglobulin (Ig) (KPL, Gaithersburg, MD), diluted 1:4,000 in dilution buffer, for $1 \mathrm{~h}$ at RT, washed 3 times, and incubated with ECL Plus Western blotting detection reagent (GE Healthcare). Results were recorded using a LAS-3000 image reader (Fujifilm, Tokyo, Japan).

\section{Immunofluorescence Analysis}

cESCs were incubated for two days on Cellware 8-well CultureSlides (BD Biosciences, San Jose, CA). Cells were fixed using PBS containing 4\% paraformaldehyde (Nacalai Tesque, Kyoto, Japan) and permeabilized with PBS containing $0.1 \%$ Triton X-100 (Sigma-Aldrich). PBS containing 3\% bovine serum albumin (BSA) (Nacalai Tesque) was added to block for $30 \mathrm{~min}$ at room temperature. An anti-chNanog pAb (1:100), an anti-Cvh mAb (1:5) and/ or an anti-GFP rabbit pAb (1:100, Clontech, Mountain View, CA) diluted with $1 \%$ BSA-PBS were added to the cells and cultured at $37^{\circ} \mathrm{C}$ for $1 \mathrm{~h}$. The secondary antibody was also diluted with $1 \%$ BSA-PBS and incubated with the cells at $37^{\circ} \mathrm{C}$ for $1 \mathrm{~h}$. To test the reactions to the secondary antibody, we used Alexa Fluor488-conjugated anti-mouse IgG antibody (1:200, Invitrogen), tetramethylrhodamine isothiocyanate (TRITC)-conjugated antirabbit IgG antibody (1:100, Southern Biotech, Birmingham, AL), Alexa Fluor594-conjugated anti-mouse IgG antibody (1:200, Invitrogen) and/or Alexa Fluor488-conjugated anti-rabbit IgG antibody (1:200, Invitrogen).

Blood containing PGCs was collected from ten chimeric embryos at stages 13 to 15 (Hamburger and Hamilton, 1951). Red blood cells were lysed and examined under fluorescent light. Stages 31 to 34 (Hamburger and Hamilton, 1951) chimera embryos were fixed using PBS containing $4 \%$ paraformaldehyde and embedded with paraffin. The sample was sliced to $5 \mu \mathrm{m}$, dewaxed and dewatered using a xylene-ethanol series. After blocking for $1 \mathrm{~h}$ using 3\% BSA-PBS, the anti-Cvh $\mathrm{mAb}(1: 5)$ and the anti-GFP pAb $(1: 100)$ was incubated overnight at $4^{\circ} \mathrm{C}$. The reaction was induced using an Alexa Fluor594-conjugated anti-mouse IgG antibody (1:200) and Alexa Fluor488-conjugated anti-rabbit IgG antibody (1:200) as a secondary antibody, and the sample was examined using a fluorescent microscope BX51 (Olympus, Tokyo, Japan,) or BZ-9700 (Keyence, Osaka, Japan).

\section{Gene Transfer}

cESCs were suspended in $700 \mu l$ OPTI-MEM (Invitrogen). A Gene Pulser XCell electroporator (Bio-Rad, Hercules, CA) was used for transfection with the following conditions: square wave, $200 \mathrm{~V}, 15 \mathrm{~ms}, 20 \mu \mathrm{g}$ linearized pCAG-EGFP-IP vector (a kind gift from Dr Niwa, CDB, RIKEN, Kobe, Japan). Selection was applied approximately $24 \mathrm{~h}$ after transfection using $0.1-0.6 \mu \mathrm{g} / \mathrm{m} l$ puromycin. Six to 14 days after selection, colonies with high 
resistance were transferred to a new plate to proliferate. Cloning of Chicken ES Cells

cESCs were treated for $2 \mathrm{~h}$ with $10 \mu \mathrm{M}$ p160-Rhoassociated coiled-coil kinase (ROCK) inhibitor (Y-27632; Wako, Osaka, Japan,) before being dissociated. PBS containing $1 \mathrm{mM}$ EGTA (Nacalai Tesque) with $0.025 \%$ trypsin (Invitrogen) was added to the cells and they were separated into single cells. They were then suspended in new CESM with Y-27632, and disseminated onto a 96 -well plate at 1 cell/well. Six to seven days after dissemination, cells were serially subcultivated into a 24-well plate. This procedure was repeated at least twice. The cloning efficiency was evaluated by counting the number of survival cESCs colonies for triplicate samples \pm the standard deviation (SD). The statistical significance (P values) in mean values of two-sample comparison was determined with Student's t-test (Microsoft Excel).

Creation of Chimera Chicken Using Chicken ES Cells

cESCs derived from BPR chickens were prepared at 5,000 cells $/ \mu l$ using DMEM containing $2 \%$ inactivated chicken serum. Two microliters of suspended cells was injected into the subgerminal cavity of a WL chicken embryo recipient. Stage $\mathrm{X}$ recipient embryos were moved into a substitute shell, which was filled with egg white and sealed with clear wrap. For the culturing procedure, we modified systems II and III devised by Rowlett and Simkiss (1987), Perry (1988), and Borwornpinyo et al. (2005). The transplanted embryo was cultured at $38.5^{\circ} \mathrm{C}$ for three days at $60 \%$ relative humidity. It was then gently moved into a new substitute shell (30-35 $\mathrm{g}$ and above), and sealed with clear wrap. The embryo was cultured at $38.5^{\circ} \mathrm{C}$ and $60 \%$ relative humidity until incubation.

\section{Results}

\section{mRNA Expression of chNanog and Cvh During Primary Culture of CBCs}

CBCs were cultured for eight days with or without rchLIF. mRNA expression of chNanog and Cvh was analyzed by RT-PCR. As shown in Figure 1, chNanog and Cvh mRNA were expressed in the presence or absence of rchLIF until the second day of culture. Expression then decreased radically after four days of culture without rchLIF, but decreased gradually and was preserved until the eighth day with rchLIF.

Characterization of Anti-chNanog and Anti-Cvh Specific Antibodies

In western blots, the anti-chNanog pAb reacted a band in nuclear extracts of $\mathrm{CBC}$, and an apparent molecular weight was approximately $40 \mathrm{kDa}$ (Fig. 2A). No chNanog band was detected in STO cells or CEF (Fig. 2a). The anti-Cvh $\mathrm{mAb}$ reacted as a band with an apparent molecular weight of approximately $75 \mathrm{kDa}$ (Fig. 2B), and did not react with somatic tissue (lung, heart, kidney and liver, data not shown). The mAb was an IgG1-k immunoglobulin and could be used in various immunological techniques including ELISA, western blotting, immunoprecipitation and immunohistochemistry.

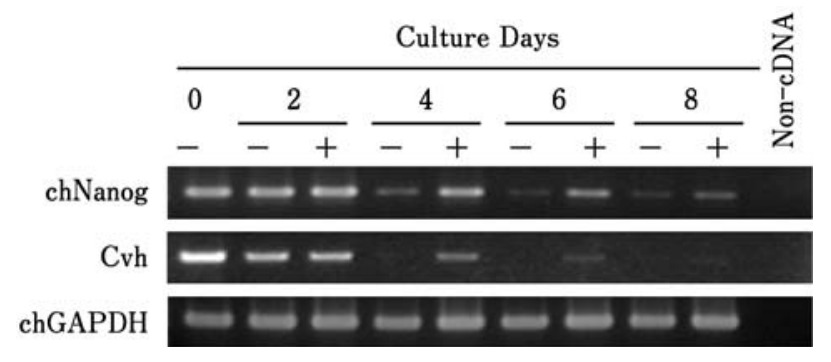

Fig. 1. Changes in chNanog and Cvh mRNA expression during CBCs culture. CBCs were cultured with $(+)$ or without $(-)$ rchLIF for eight days. mRNA was isolated for RT-PCR using chNanog, Cvh or chGAPDH gene specific primers. chGAPDH gene amplification products were used as an internal control.
A

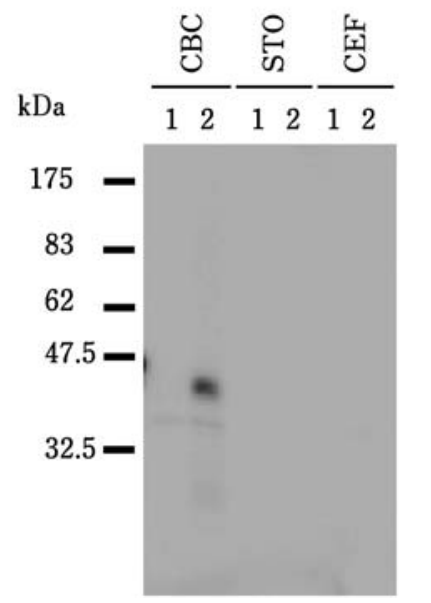

B

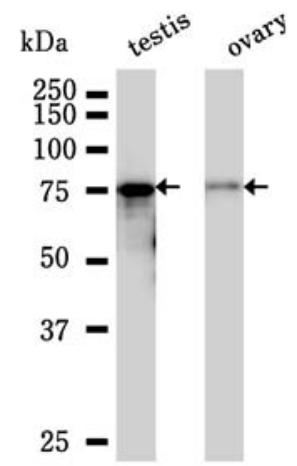

Fig. 2. Characterization of anti-chNanog and anti-Cvh specific antibodies. (A) The cytoplasmic fractions (1) and nuclear fractions (2) from CBCs, STO cells and CEF were separated on a $10 \%$ SDS-PAGE gel and analyzed by western blotting using the anti-chNanog pAb. (B) Western blot analysis with newly hatched chick testis and ovary lysates using the anti-Cvh $\mathrm{mAb}$. The relative mobility of protein markers is indicated on the left.

\section{Long-term Culture of CBCs and Their Characteristics}

CBCs obtained from one egg were cultured on STO cells feeder cells using CECM with rchLIF. The growth morphology of CBCs was similar to primate ES cells and consisted of a circular colony with a clear contour, and each individual cell visible (Fig. 3-2a). Cells had to be subcultured every second or third day to maintain their proliferative potential. After cryopreservation, cells maintained the same morphology when recultured (data not shown).

Using CBCs that were successively subcultured for 100 days or more, we analyzed the expression of chNanog and Cvh mRNA. As shown in Figure 3-1, we confirmed ex- 
1

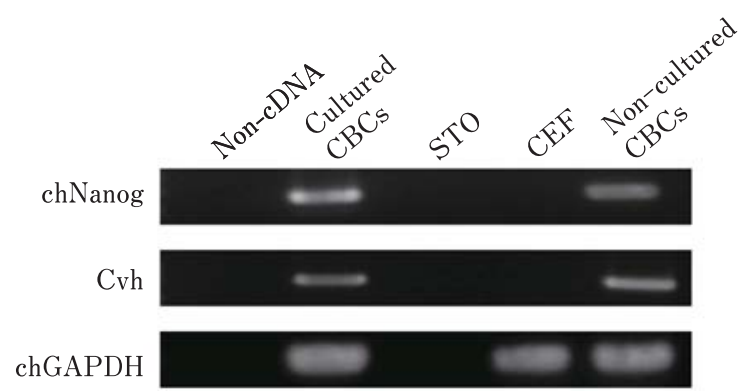

2

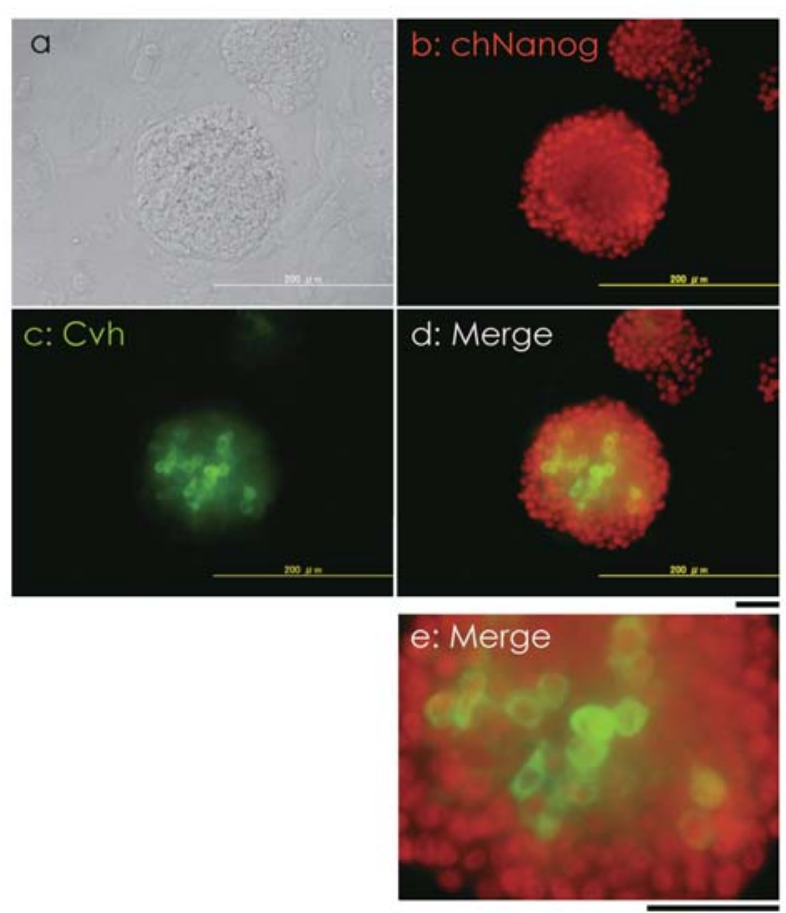

Fig. 3. Characterization of long-term cultured CBCs. (1) Expression analysis of chNanog and Cvh mRNA. RNA was isolated from CBCs successively subcultured for 212 days and used for RT-PCR with chNanog, Cvh and chGAPDH gene specific primers. RNA isolated from STO cells, CEF and non-cultured CBCs was also examined with the same primers. (2) (a) A circular colony of cultured CBCs. Immunofluorescence analysis of chNanog (b) and Cvh (c) protein. CBCs successively subcultured for 105 days were tested with double fluorescent dye staining using anti-chNanog pAb and anti-Cvh mAb. (d) Double immunofluorescence imaging. (e) High magnification. Scale bar $=50 \mu \mathrm{m}$.

pression of chNanog and Cvh mRNA immediately after collection of the CBCs. As shown in Figure 3-2, chNanog was detected in the nucleus of most cells that formed a colony (Fig. 3-2b), while Cvh expression was confirmed in the cytoplasm of cells that existed around the central area of the colony (Fig. 3-2c). The cultured CBCs showed a capacity for chimeric formation during a transplant experiment that used a fertilized egg derived from WL
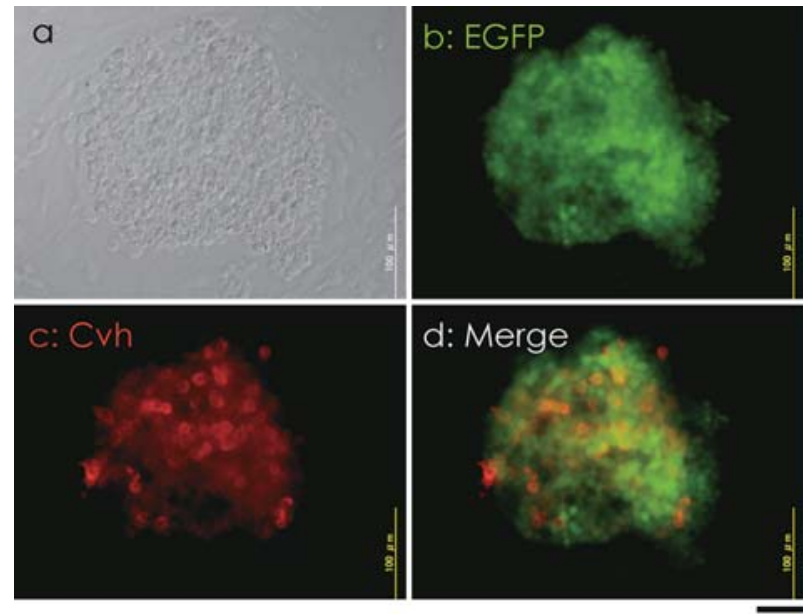

Fig. 4. Characterization of EGFP-cESCs. Anti-Cvh $\mathrm{mAb}$ fluorescence was examined using EGFP-cESCs. (a) A circular colony of EGFP-cESCs. (b) Expression of EGFP. (c) Immunofluorescence analysis of Cvh protein. (d) Double fluorescence imaging. Scale bar $=$ $50 \mu \mathrm{m}$.

chickens as a recipient (data not shown). We therefore named the cultured CBCs cESCs and used them for the following study. While CBCs used in this study were all collected from fertilized eggs derived from BPR chickens, it is also possible to establish cESCs with the same characteristics from CBCs derived from WL or Bovans Nera chickens using the same culture methods.

\section{Characteristics of Chicken ES Cells Harboring the EGFP Gene}

In order to create genetically-modified chickens using established cESCs, it was deemed necessary to preserve the expression of chNanog and Cvh even after gene transfer. Moreover, the transfer of reporter genes such as enhanced green fluorescent protein (EGFP) gene into cESCs enables their differentiation into germ cells to be traced. We electroporated linearized pCAG-EGFP-IP carrying the EGFP and puromycin resistance genes into cESCs and performed selection with puromycin. After 6-14 days, we obtained puromycin-resistant cESCs that proliferated by forming a circular colony like pre-transfer cells (Fig. 4a). Expression of EGFP was detected in almost all cells that formed a colony (Fig. 4b). Immunofluorescent staining with anti-Cvh mAb confirmed expression of Cvh (Fig. 4c, d) after gene transfer. In the same way, chNanog was expressed in the gene-transfer cESCs (data not shown).

Cell Cloning of Chicken ES Cells and Characteristics of Cloned EGFP-cESCs

Cell cloning is essential in homogenizing the areas of gene insertion of gene-transfer cESCs. Moreover, it may also be possible to induce only Cvh-positive cESCs to proliferate. We therefore tried to clone cESCs transfected with EGFP (EGFP-cESCs) by limiting dilution. Three kinds of EGFP-cESCs (\#1, 2 and 3) derived from differ- 


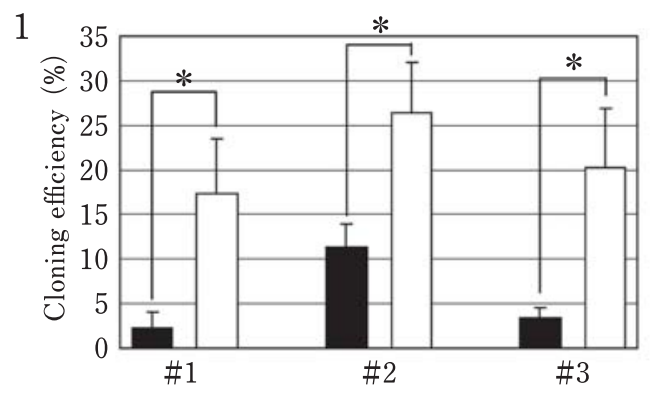

2
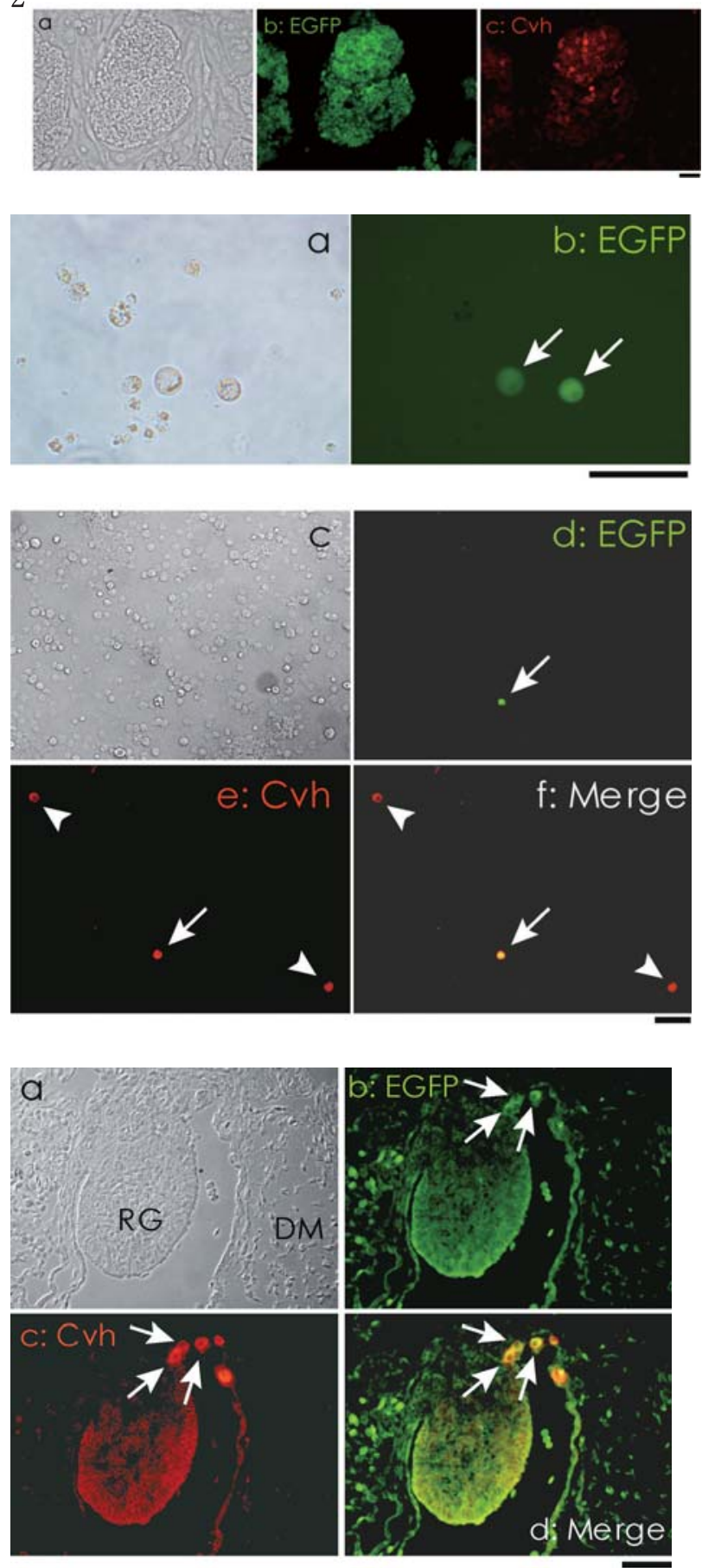

Fig. 5. Cloning efficiency of EGFP-cESCs and Cvhexpression of cloned EGFP-cESCs. (1) Cloning efficiency of EGFP-cESCs (\#1, 2 and 3), with (white bar) and without Y-27632 (black bar) $(*, p<0.05$ versus control, $n=3$ ). (2) Expression of Cvh protein analyzed by immunofluorescence staining using anti-Cvh mAb. (a) A circular colony of EGFP-cESCs. (b) Expression of EGFP. (c) Immunofluorescence analysis of Cvh protein. Scale bar $=50 \mu \mathrm{m}$.

Fig. 6. EGFP expression in migrating PGCs. (a) Whole blood was collected from stages $13-15$ chimeric embryos. (b) Living cells were observed directly under a fluorescent microscope. Arrows indicate EGFPpositive cells. Scale bar $=50 \mu \mathrm{m}$. (c) Smear sample of embryo blood collected from 2.5-day incubated embryos. (d) Expression of EGFP. (e) Immunofluorescence analysis of $\mathrm{Cvh}$ protein. Arrowheads indicate recipient PGCs (Cvh-positive cells). (f) Double fluorescence imaging. Arrow indicates PGC derived from cloned EGFP-cESCs among Cvh-positive cells. Scale bar $=50 \mu \mathrm{m}$.

Fig. 7. EGFP expression into transverse section of a sixth day incubated embryo. Anti-Cvh mAb fluorescence was examined using a tissue slice (a) near the gonadal ridge of a sixth day old incubated chimera embryo. (b) Expression of EGFP. (c) Immunofluorescence analysis of Cvh protein. (d) Double immunofluorescence imaging. Arrows indicate PGC derived from cloned EGFP-cESCs among PGC (Cvh-positive cells). DM; dorsal mesentery, RG; right gonad. Scale bar $=50 \mu \mathrm{m}$. 
ent eggs were disseminated into 96-well plates at 1 cell/ well. Only cells that formed a colony were counted. In cases where limiting dilution was performed only with CESM, the cloning efficiency was $15 \%$ or lower. In many cases, cells were destroyed during successive subcultures. When Y-27632 was added to CESM, the cloning efficiency increased significantly to $15 \%$ or higher (Fig. $5-1, p<$ 0.05 ). Subsequent successive subculture was also successful. In immunofluorescence staining, cloned EGFPcESCs was all EGFP- and chNanog-positive cells that formed colony (Fig. 5-2a, b). Some of these EGFPexpressing cells were also Cvh-positive (Fig. 5-2c). By repeating this method more than twice, we acquired cloned EGFP-cESCs that were used for the following study.

EGFP Expression in PGCs and Capacity of Cloned EGFPcESCs to Form a Chimera

Based on the above findings, we transplanted cloned EGFP-cESCs to a recipient embryo of a fertilized egg derived from WL chickens. In collecting stages 13 to 15 embryo blood, living EGFP-positive cells were shown to have the morphological characteristics of PGCs (Fig. 6a, b). In immunofluorescence staining using smear of embryo blood cells, we confirmed expression of EGFP deriving from cloned EGFP-cESCs among Cvh-positive cells (Fig. 6c-f). Tissues near the gonadal ridge of an incubated egg in its sixth day revealed expression of EGFP among Cvh-positive cells, migrating through the dorsal intestinal membrane to the gonadal ridge (Fig. 7a-d). In its eighth day, some EGFP-positive cells were observed among many Cvh-positive cells in the gonadal ridge (data not shown). This indicated that Cvh-positive cells among cloned EGFP-cESCs migrate into the gonadal ridge.

Expression of EGFP was observed all over the transplanted embryo on its fifth day of incubation, indicating that cloned EGFP-cESCs possess the capacity for pluripotency (Fig. 8a, b). Continuation of the incubation showed the transplanted embryo to develop normally, and we observed the hatching of a chimera chicken with black feathers, which is an expression of BPR chickens (Fig. 8c, d).

\section{Discussion}

In 2006, van de Lavoir and others reported the establishment of cESCs that differentiated into somatic cells at a high frequency (2006a). However, these ES cells could not differentiate into germ cells and were unable to maintain their potential for germline transmisson. Subsequent research has found that these established ES cells contained very few Cvh-positive cells (2006b). Unlike mammals, stage $\mathrm{X}$ CBCs contain approximately $30 \mathrm{Cvh}$ positive cells $(0.1 \%)$ that are thought to be precursor PGCs (Stepinska and Olszanska, 1983; Tsunekawa et al., 2000). Many researchers have shown that it is possible to create germline chimera chickens by transplanting cells from these CBCs to a recipient embryo (Petitte et al., 1990; Kagami et al., 1995).
To establish cESCs from CBCs that will contribute to germ cells, it is considered essential to develop a culture method that can preserve the characteristics of stage $\mathrm{X}$ $\mathrm{CBCs}$, in other words to preserve Cvh-positive cells (Lavial et al., 2009). This study confirmed that it is possible to maintain expression of chNanog and Cvh mRNA by using rchLIF in the primary culture of CBCs (Fig. 1). Although this expression gradually decreases after six days of culture, we found that successive subcultures performed within one to three days enabled long-term culture and expression of $\mathrm{Cvh}$. In the case of $\mathrm{CBC}$ culture using CESM without rchLIF, the expression of chNanog and Cvh mRNA decreased extremely (Fig. 1). Yamashita and others reported that chLIF mRNA expression decreased extremely after 3 days of $\mathrm{CBC}$ culture, and suggested that CBCs themselves produced chLIF in culture, which acted as an autocrine signal (2006). These results suggest that the expression of chNanog and Cvh mRNA is maintained for a short culture time by autocrine signal of chLIF. The effect of LIF on the cESCs established in this study produced characteristics that were closer to those of mouse ES cells than primate ES cells (Niwa et al., 1998; Smith et al., 1988; Thomson and Marshall, 1998; Matsuda et al., 1999; Suemori et al., 2001; Horiuchi et al., 2004; Yamashita et al., 2006). However, the tendency of cESCs to be destroyed when the colony or its morphology is separated into single cells shows a close resemblance to primate ES cells.

The biggest advantage of creating genetically-modified animals from ES cells is that gene targeting by recombining homologous genes is made possible (Thomas and Capecchi, 1987; Mansour et al., 1988). However, while cell cloning is an essential technology to achieve this, the ready destruction of cESCs due to colony dissociation makes the incorporation of this difficult. In 2007, Watanabe and others reported that the addition of ROCK inhibitor was effective in culturing human ES cells from single cells (2007). Therefore, we used ROCK inhibitor for the cloning of cESCs in this study and effectively cultured and cloned cESCs from single cells (Fig. 5).

We next hypothesized that it might be possible to clone only Cvh-positive cESCs. However, not all Cvh-positive cells turned into cloned cESCs but were scattered inside the colony, rather like pre-cloned cells. This may have resulted from the fact that only Cvh-positive cells showed clonal proliferation during the cloning process while others lost expression of Cvh. Alternatively, the culture method established in this study might have caused some of the cESCs to become Cvh-positive. Whether it is true or not, it is certain that Cvh-positive cells among our established cESCs keep proliferating in vitro (Fig. 3, 4, 52 ). We aim to use our culture system to determine the development of PGCs among birds.

By transferring the EGFP gene into cESCs, we were able to trace expression of EGFP to PGCs in the migration stage (Fig. 6, 7). In immunostaining of the gonadal ridge, the whole gonad was seen green (Fig. 7). The rea- 

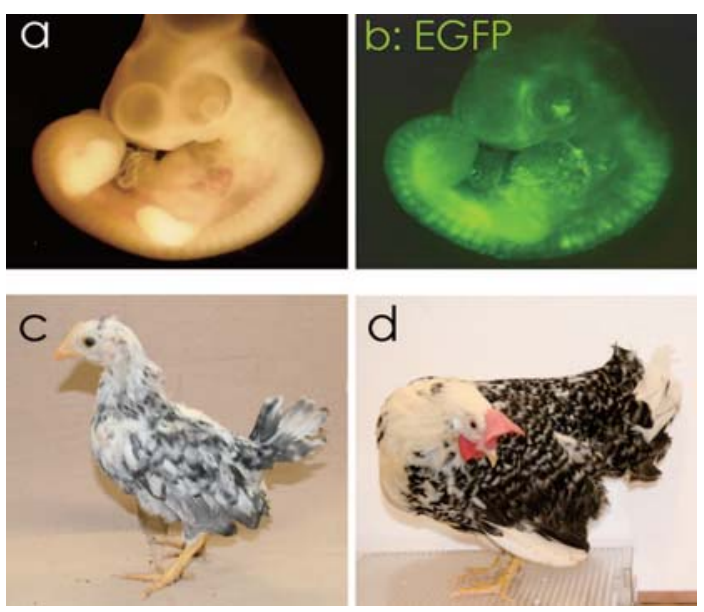

Fig. 8. Capacity of cloned EGFP-cESCs to form a chimera. EGFP-cESCs transplanted to a stage $\mathrm{X}$ recipient embryo of WL chickens were examined for their capacity to form chimeras. (a, b) Expression of EGFP was observed by using the transplanted embryo on its fifth day of incubation. A chimeric chicken with black feathers while is an expression of BPR chickens. (c) Forty days old. (d) Six-month old.

son for this result should be that the cESCs frequently contributed the somatic cells of the gonad. We should transplant only Cvh-positive cells among EGFP-positive cells to a recipient embryo for the future. More recently, in preliminary results, we have detected EGFP gene in sperm genomes and EGFP-expression in oocytes of chimeric chickens (our unpublished data, 2010), and are confident in creating genetically-modified chickens using cESCs established in the present study.

In conclusion, we show the derivation of cESCs from CBCs cultured with chLIF. The cESCs express chNanog and $\mathrm{Cvh}$ and are capable of long-term successive subculture, after gene transfer and cell cloning. They can also migrate into gonadal ridge and can be transplanted into recipient embryos for the development of a chicken chimera. These results indicate that novel cESCs have the potential of creating genetically-modified chickens.

\section{Acknowledgments}

The authors thank Dr Hitoshi Niwa for kindly donating the pCAG-EGFP-IP vector and Dr Hirofumi Suemori for valuable discussion. This work was supported by a Grantin-Aid for Scientific Research (B) (No. 16580279) from the Ministry of Education, Culture, Sports, Science and Technology of Japan, the Biotechnology Cluster in Central Hiroshima, Hiroshima Prefecture, Japan, and Research Fellowships of the Japan Society for the Promotion of Science for Young Scientists.

\section{References}

Adachi K, Kawase E, Yasuchika K, Sumi T, Nakatsuji N and Suemori H. Establishment of the gene-inducible system in primate embryonic stem cell lines. Stem Cells, 24: 25662572. 2006.

Borwornpinyo S, Brake J, Mozdziak PE and Petitte JN. Culture of chicken embryos in surrogate eggshells. Poultry Science, 84: 1477-1482. 2005.

Buehr M, Meek S, Blair K, Yang J, Ure J, Silva J, McLay R, Hall J, Ying QL and Smith A. Capture of authentic embryonic stem cells from rat blastocysts. Cell, 135: 1287-1298. 2008.

Evans MJ and Kaufman MH. Establishment in culture of pluripotential cells from mouse embryos. Nature, 292: 154156. 1981.

Eyal-Giladi $\mathrm{H}$ and Kochav S. From cleavage to primitive streak formation: a complementary normal table and a new look at the first stages of the development of the chick. I. General morphology. Developmental Biology, 49: 321-337. 1976.

Galfre G, Howe SC, Milstein C, Butcher GW and Howard JC. Antibodies to major histocompatibility antigens produced by hybrid cell lines. Nature, 266: 550-552. 1977.

Hamburger V and Hamilton HL. A series of normal stages in the development of the chick embryo. Journal of Morphology, 88: 49-92. 1951. (reprinted, Developmental Dynamics 195: 231-272. 1992.)

Horiuchi H, Tategaki A, Yamashita Y, Hisamatsu H, Ogawa M, Noguchi T, Aosasa M, Kawashima T, Akita S, Nishimichi N, Mitsui N, Furusawa S and Matsuda H. Chicken leukemia inhibitory factor maintains chicken embryonic stem cells in the undifferentiated state. The Journal of Biological Chemistry, 279: 24514-24520. 2004.

Horiuchi H, Furusawa S and Matsuda H. Maintenance of chicken embryonic stem cells in vitro. Methods in Molecular Biology, 329: 17-34. 2006.

Kagami H, Clark ME, Verrinder Gbbins AM and Etches RJ. Sexual differentiation of chimeric chickens containing ZZ and ZW cells in the germline. Molecular Reproduction and Development, 42: 379-387. 1995.

Lavial F, Acloque H, Bachelard E, Nieto MA, Samarut J and Pain B. Ectopic expression of Cvh (Chicken vasa homologue) mediates the reprogramming of Chicken Embryonic Stem cells to a germ cell fate. Developmental Biology, 330: 73-82. 2009.

Leighton PA, van de Lavoir MC, Diamond JH, Xia C and Etches RJ. Genetic modification of primordial germ cells by gene trapping, gene targeting, and phiC31 integrase. Molecular Reproduction and Development, 75: 1163-1175. 2008.

Li P, Tong C, Mehrian-Shai R, Jia L, Wu N, Yan Y, Maxson RE, Schulze EN, Song H, Hsieh CL, Pera MF and Ying QL. Germline competent embryonic stem cells derived from rat blastocysts. Cell, 135: 1299-1310. 2008.

Mansour SL, Thomas KR and Capecchi MR. Disruption of the proto-oncogene int-2 in mouse embryo-derived stem cells: a general strategy for targeting mutations to non-selectable genes. Nature, 336: 348-352. 1988.

Martin GR. Isolation of a pluripotent cell line from early mouse embryos cultured in medium conditioned by teratocarcinoma stem cells. Proceedings of the National Academy of Sciences of the United States of America, 78: 7634-7638. 1981.

Matsuda T, Nakamura T, Nakao K, Arai T, Katsuki M, Heike T and Yokota T. STAT3 activation is sufficient to maintain an undifferentiated state of mouse embryonic stem cells. EMBO Journal, 18: 4261-4269. 1999.

Nichols J, Evans EP and Smith AG. Establishment of germ-line- 
competent embryonic stem (ES) cells using differentiation inhibiting activity. Development, 110: 1341-1348. 1990.

Niwa H, Burdon T, Chambers I and Smith A. Self-renewal of pluripotent embryonic stem cells is mediated via activation of STAT3. Genes and Development, 12: 2048-2060. 1998.

Ogura $\mathrm{H}$ and Fujiwara T. Establishment and characterization of a virus-free chick cell line. Acta Medica Okayama, 41: 141143. 1987.

Pain B, Clark ME, Shen M, Nakazawa H, Sakurai M, Samarut J and Etches RJ. Long-term in vitro culture and characterisation of avian embryonic stem cells with multiple morphogenetic potentialities. Development, 122: 2339-2348. 1996.

Perry MM. A complete culture system for the chick embryo. Nature, 331: 70-72. 1988.

Petitte JN, Clark ME, Liu G, Verrinder Gibbins AM and Etches RJ. Production of somatic and germline chimeras in the chicken by transfer of early blastodermal cells. Development, 108: 185-189. 1990.

Rowlett KA and Simkiss K. Explanted embryo culture: in vitro and in ovo techniques for domestic fowl. British Poultry Science, 28: 91-101. 1987.

Shulman M, Wilde CD and Kohler G. A better cell line for making hybridomas secreting specific antibodies. Nature, 276: 269-270. 1978.

Smith AG, Heath JK, Donaldson DD, Wong GG, Moreau J, Stahi $\mathrm{M}$ and Rogers D. Inhibition of pluripotential embryonic stem cell differentiation by purified polypeptides. Nature, 336: 688-690. 1988.

Stepinska U and Olszanska B. Cell multiplication and blastoderm development in relation to egg envelope formation during uterine development of quail (Coturnix coturnix japonica) embryo. The Journal of Experimental Zoology, 228: 505510. 1983.

Suemori H, Tada T, Torii R, Hosoi Y, Kobayashi K, Imahie H, Kondo Y, Iritani A and Nakatsuji N. Establishment of embryonic stem cell lines from cynomolgus monkey blastocysts produced by IVF or ICSI. Developmental Dynamics, 222: 273-279. 2001.

Tajima A, Naito M, Yasuda Y and Kuwana T. Production of germ line chimera by transfer of primordial germ cells in the domestic chicken (Gallus domesticus). Theriogenology, 40: 509-519. 1993.
Teramura T, Takahara T, Kawata N, Fujinami N, Mitani T, Takenoshita M, Matsumoto K, Saeki K, Iritani A, Sagawa $\mathrm{N}$ and Hosoi Y. Primate embryonic stem cells proceed to early gametogenesis in vitro. Cloning and Stem Cells, 9: 144 -156. 2007.

Thomas KR and Capecchi MR. Site-directed mutagenesis by gene targeting in mouse embryo-derived stem cells. Cell, 51: 503-512. 1987.

Thomson JA, Itskovitz-Eldor J, Shapiro SS, Waknitz MA, Swiergiel JJ, Marshall VS and Jones JM. Embryonic stem cell lines derived from human blastocysts. Science, 282: 1145-1147. 1998.

Thomson JA and Marshall VS. Primate embryonic stem cells. Current Topics in Developmental Biology, 38: 133-165. 1998.

Tsunekawa N, Naito M, Sakai Y, Nishida T and Noce T. Isolation of chicken vasa homolog gene and tracing the origin of primordial germ cells. Development, 127: 2741-2750. 2000.

van de Lavoir MC, Mather-Love C, Leighton P, Diamond JH, Heyer BS, Roberts R, Zhu L, Winters-Digiacinto P, Kerchner A, Gessaro T, Swanberg S, Delany ME and Etches RJ. High-grade transgenic somatic chimeras from chicken embryonic stem cells. Mechanism of Development, 123: 31-41. 2006a.

van de Lavoir MC, Diamond JH, Leighton PA, Mather-Love C, Heyer BS, Bradshaw R, Kerchner A, Hooi LT, Gessaro TM, Swanberg SE, Delany ME and Etches RJ. Germline transmission of genetically modified primordial germ cells. Nature, 441: 766-769. 2006b.

Watanabe K, Ueno M, Kamiya D, Nishiyama A, Matsumura M, Wataya T, Takahashi JB, Nishikawa S, Nishikawa S, Muguruma K and Sasai Y. A ROCK inhibitor permits survival of dissociated human embryonic stem cells. Nature Biotechnology, 25: 681-686. 2007.

Yamashita Y, Tategaki A, Ogawa M, Horiuchi H, Nishida K, Akita S, Matsuda $\mathrm{H}$ and Furusawa S. Effect of novel monoclonal antibodies on LIF-induced signaling in chicken blastodermal cells. Developmental and Comparative Immunology, 30: 513-522. 2006.

Yamauchi K, Hasegawa K, Chuma S, Nakatsuji N and Suemori $\mathrm{H}$. In vitro germ cell differentiation from cynomolgus monkey embryonic stem cells. PLoS One, 4; e5338. 2009. 